03,13

\title{
Метод управления полярностью слоев GaN при эпитаксиальном синтезе GaN/AIN гетероструктур на гибридных подложках $\mathrm{SiC} / \mathrm{Si}$
}

\author{
(C) А.М. Мизеров ${ }^{1}$, С.А. Кукушкин ${ }^{2}$, Ш.Ш. Шарофидинов ${ }^{3}$, А.В. Осипов ${ }^{4}$, С.Н. Тимошнев ${ }^{1}$, \\ К.Ю. Шубина ${ }^{1}$, Т.Н. Березовская ${ }^{1}$, Д.В. Мохов ${ }^{1}$, А.Д. Буравлев ${ }^{1,3}$ \\ ${ }^{1}$ Санкт-Петербургский национальный исследовательский Академический университет РАН, \\ Санкт-Петербург, Россия \\ ${ }^{2}$ Институт проблем машиноведения РАН, \\ Санкт-Петербург, Россия \\ ${ }^{3}$ Физико-технический институт им. А.Ф. Иофрфе РАН, \\ Санкт-Петербург, Россия \\ ${ }^{4}$ Санкт-Петербургский национальный исследовательский университет \\ информационных технологий, механики и оптики, \\ Санкт-Петербург, Россия \\ E-mail: andreymizerov@rambler.ru
}

Поступила в Редакцию 16 июля 2019 г.

В окончательной редакции 16 июля 2019 г.

Принята к публикации 25 июля 2019 г.

Обнаружен эффект инверсии полярности в слоях $\mathrm{GaN}$ с N-полярного слоя $\mathrm{GaN}$ на Ga-полярный слой $\mathrm{GaN}$ в процессе последовательного выращивания пленок $\mathrm{GaN}$ на гибридных подложках $\mathrm{SiC} / \mathrm{Si}(111)$ методами методом молекулярно-пучковой эпитаксии с плазменной активацией азота и методом хлорид-гидридной эпитаксии. Разработан новый метод формирования свободных от трещин Ga-полярных гетероструктур $\mathrm{GaN} / \mathrm{AlN}$ на гибридных подложках $\mathrm{SiC} / \mathrm{Si}(111)$. Метод включает в себя два этапа выращивания слоев $\mathrm{GaN}$. На первом этапе методом МПЭ ПА на поверхности $\mathrm{SiC} / \mathrm{Si}(111)$ выращивается переходный N-полярный слой GaN. На втором этапе, методом ХГЭ на полученном N-полярном слое GaN выращиваются два слоя, а именно слой $\mathrm{AlN}$, а затем слой $\mathrm{GaN}$, который на этом этапе вырастает Ga-полярной ориентации. Установлено, что травление в растворе $\mathrm{KOH}$ затрагивает только N-полярный переходный слой $\mathrm{GaN}$ и приводит к полному его удалению, что позволят полностью отделить основной $\mathrm{Ga}$-полярный слой $\mathrm{GaN}$ от подложки $\mathrm{SiC} / \mathrm{Si}(111)$. Метод позволяет выращивать свободные от трещин и упруго не напряженные толстые слои $\mathrm{GaN}$, и переносить их на подложки других материалов.

Ключевые слова: $\mathrm{GaN}$, $\mathrm{AlN}$, кремний, $\mathrm{SiC}$ на $\mathrm{Si}$, метод замещения атомов, молекулярно-пучковая эпитаксия с плазменной активацией, хлорид-гидридная эпитаксия.

DOI: $10.21883 /$ FTT.2019.12.48535.06ks

\section{1. Введение}

Уникальные физические свойства широкозонных полупроводниковых соединений на основе $\mathrm{GaN}$ делают эти материалы перспективными для создания современных электронных приборов и обуславливают большой интерес к исследованиям эпитаксиального синтеза этих материалов [1-4].

Для эпитаксиального синтеза пленок $\mathrm{GaN}$, как правило, используют рассогласованные по параметрам решеток c GaN монокристаллические подложки, например, сапфира $\left(c-\mathrm{Al}_{2} \mathrm{O}_{3}\right)$ или кремния $(\mathrm{Si})$. В результате в процессе роста $\mathrm{GaN}$ на гетерогранице пленка-подложка образуется большое число различных дефектов, в частности дислокаций несоответствия. Использование монокристаллических подложек карбида кремния ( $\mathrm{SiC})$, параметры решетки которых имеют более близкие к параметрам решеток $\mathrm{AlN}$ и $\mathrm{GaN}$ значения, для роста слоев $\mathrm{GaN}$ приводят к значительно лучшим результатам. Однако, подложки $\mathrm{SiC}$ пока еще весьма дороги, отделение, при необходимости, от них слоев $\mathrm{GaN}$ весьма не простая задача и, наконец, самое главное, современные задачи микро и оптоэлектроники требуют симбиоза свойств кремния со свойствами широкозонных полупроводников.

Таким образом, одной из ключевых проблем, сдерживающей развитие технологии синтеза высококачественных $\mathrm{GaN}$ соединений является как отсутствие экономически доступных монокристаллических $\mathrm{GaN}$ подложек высокого качества и больших размеров, так и не достаточное, на сегодняшний день развитее технологии выращивания слоев $\mathrm{GaN}$ на кремниевой подложке. Решению этой проблемы в настоящее время уделяется огромное внимание. Большое внимание уделяется и проблеме отделения синтезированных эпитаксиальных объемных слоев $\mathrm{GaN}$ от упомянутых выше сильно рассогласованных с $\mathrm{GaN}$ по параметрам решеток подложек [5-8]. Следует отметить, что преимущество использования для эпитаксии объемных слоев $\mathrm{GaN}$ подложек кремния обусловлено экономической доступностью пластин монокристаллического кремния высокого качества с диа- 
метром вплоть до $450 \mathrm{~mm}$ о которой было указано выше, отсутствием полярности у подложек $\mathrm{Si}$, что позволяет выращивать на них как Ga-, так и $\mathrm{N}$-полярные слои $\mathrm{GaN}[9]$, а также возможностью отделения слоев $\mathrm{GaN}[8]$.

В работе [8] была показана потенциальная возможность менять полярность слоев $\mathrm{GaN}$ с азотной на галлиевую при их росте на подложках $\mathrm{Si}(111)$. Для этого используют такие эпитаксиальных технологии как: молекулярно-пучковая эпитаксия с плазменной активацией азота (МПЭ ПА) при которой растут на $\mathrm{Si}(111)$ подложках $\mathrm{N}$-полярные переходные слои $\mathrm{GaN}$ и метод газофазной эпитаксии из металлоорганических соединений (ГФЭМОС) при использовании которого на $\mathrm{Si}(111)$ подложках растут $\mathrm{Ga}$-полярные объемные слои $\mathrm{GaN}$. Кроме того, в работе [8] была показана потенциальная возможность отделения верхнего Ga-полярного слоя $\mathrm{GaN}$ от подложки $\mathrm{Si}(111)$ посредством вытравливания промежуточного $\mathrm{N}$-полярного слоя $\mathrm{GaN}$ в растворе $\mathrm{KOH}$.

Ранее в работах $[10,11]$ мы показали, что слои $\mathrm{GaN}$, выращенные методом МПЭ ПА на решеточно согласованных гибридных подложках $\mathrm{SiC} / \mathrm{Si}(111)$, полученных методом замещения атомов, обладают более высоким качеством, по сравнению с аналогичными слоями, выращенными на подложках $\mathrm{Si}(111)$. В работах $[9,10]$ было показано, что для роста $\mathrm{N}$-полярных слоев $\mathrm{GaN}$ на подложках $\mathrm{SiC} / \mathrm{Si}(111)$ необходимо использовать стехиометрические соотношения между потоками $\mathrm{Ga}$ и $\mathrm{N}$ на начальных стадиях роста $\mathrm{GaN}$. В тоже время, для того, чтобы выросли $\mathrm{N}$-полярные слои $\mathrm{GaN}$ на подложках $\mathrm{Si}(111)$ необходимо либо использовать большие потоки активированного азота, либо осуществлять рост при меньших температурах подложки. Однако, последнее обстоятельство приводит низкому качеству синтезируемых слоев $\mathrm{GaN}$. Известно, что использование подложек $\mathrm{SiC} / \mathrm{Si}(111)$ для роста слоев $\mathrm{GaN}$ методом хлорид-гидридной эпитаксии (ХГЭ) позволяет получать толстые, свободные от трещин слои $\mathrm{GaN}$ относительно высокого качества [12].

В настоящей работе изложены основные технологические идеи и приемы нового метода получения толстых, отделенных от подложки $\mathrm{Si} \mathrm{Ga}-$ полярных слоев $\mathrm{GaN}$, выращенных методом ХГЭ на $\mathrm{N}$-полярных переходных слоях $\mathrm{GaN}$, сформированных методом МПЭ ПА на подложках $\mathrm{SiC} / \mathrm{Si}(111)$.

\section{2. Эксперимент}

Для роста нелегированных слоев $\mathrm{GaN}$ использовали трехдюймовые подложки $\mathrm{Si} p$-типа проводимости ориентации (111) на которых предварительно была синтезирована наномасштабная пленка $\mathrm{SiC}$ методом [13-16].

После процедуры синтеза пленки $\mathrm{SiC}$ полученные гибридные подложки $\mathrm{SiC} / \mathrm{Si}(111)$, которые в дальнейшем будут удалены, поэтому мы будем их назвать временными (или виртуальными подложками) проходили процедуру химической очистки от органических загрязнений, включающую кипячение в четыреххлористом углероде и изопропаноле. Сразу после окончания процедуры химической очистки эти подложки помещались в загрузочную камеру установки МПЭ ПА промышленного типа Veeco Gen 200, где отжигались в течение двух h в условиях сверхвысокого вакуума $\left(\sim 10^{-8}\right.$ Torr $)$ при температуре $\sim 200^{\circ} \mathrm{C}$ с целью удаления паров воды с поверхности $\mathrm{SiC}$.

После окончания процедуры предварительного отжига подложки перемещались в ростовую камеру установки МПЭ ПА, где проходили процедуру высокотемпературного отжига, проводимого в течение $30 \min$ при $T_{S} \sim 850^{\circ} \mathrm{C}$ в потоке активированного азота $F_{\mathrm{N}} \sim 0.1 \mu \mathrm{m} / \mathrm{h}$ с целью очистки $\mathrm{SiC}$ поверхностей от оставшихся на поверхности инородных атомов.

После этого осуществлялся МПЭ ПА синтез переходных слоев $\mathrm{GaN}$ на подложках $\mathrm{SiC} / \mathrm{Si}(111)$, который состоял из двух стадий: сначала выращивался зародышевый низкотемпературный слой LT-GaN c толщиной $15 \mathrm{~nm}$ при $T_{S}=650^{\circ} \mathrm{C}$ и единичных соотношениях потоков галлия и активированного азота $F_{\mathrm{Ga}}=F_{\mathrm{N}} \sim 0.1 \mu \mathrm{m} / \mathrm{h}$. Затем выращивался более высокотемпературный слой $\mathrm{GaN}$ с толщиной $\sim 750 \mathrm{~nm}$ при $T_{S}=730^{\circ} \mathrm{C}$ и соотношении потоков $F_{\mathrm{Ga}} / F_{\mathrm{N}} \sim 6$. Для in situ наблюдения за зарождением и изменением морфологии поверхности переходных слоев $\mathrm{GaN}$ использовался метод дифракции отраженных быстрых электронов (ДОБЭ).

После МПЭ ПА синтеза переходного слоя GaN полученная структура $\mathrm{GaN} / \mathrm{SiC} / \mathrm{Si}(111)$ была разделена на три части. Одна часть структуры была исследована на предмет определения полярности переходного слоя $\mathrm{GaN}$ с помощью разработанной методики травления образцов в КОН [9]. Вторая часть выращенной структуры $\mathrm{GaN} / \mathrm{SiC} / \mathrm{Si}(111)$ была загружена в зону реактора установки ХГЭ для синтеза основного слоя $\mathrm{GaN}$ с толщиной порядка $\sim 3 \mathrm{mkm}$ непосредственно на $\mathrm{GaN}$ поверхности структуры $\mathrm{GaN} / \mathrm{SiC} / \mathrm{Si}(111)$. В процессе ХГЭ синтеза $\mathrm{GaN}$ температура в зоне роста составляла $T=1050^{\circ} \mathrm{C}$ поток $\mathrm{NH}_{3}$ и $\mathrm{HCl}$ составляли $V_{\mathrm{NH}_{3}} \sim 1.5 \mathrm{ml} / \mathrm{min}$ и $V_{\mathrm{HCl}} \sim 200 \mathrm{ml} / \mathrm{min}$, соответственно. Третья часть структуры $\mathrm{GaN} / \mathrm{SiC} / \mathrm{Si}(111)$ была использована для роста основного слоя $\mathrm{GaN}$ методом ХГЭ, проводимого в два этапа. На первом этапе ХГЭ на $\mathrm{GaN}$ поверхности структуры $\mathrm{GaN} / \mathrm{SiC} / \mathrm{Si}(111)$ осуществлялся рост промежуточного слоя с AlN с толщиной $\sim 280 \mathrm{~nm}$ при $T=1080^{\circ} \mathrm{C}, \quad V_{\mathrm{NH}_{3}} \sim 11 / \mathrm{min}$ и $V_{\mathrm{HCl}} \sim 1.7 \cdot 10^{-6} \mathrm{ml} / \mathrm{min}$. После этого растился основной слой $\mathrm{GaN}$ с толщиной порядка $\sim 3 \mu \mathrm{m}$ при $T=1050^{\circ} \mathrm{C}, V_{\mathrm{NH}_{3}} \sim 1.5 \mathrm{ml} / \mathrm{min}$ и $V_{\mathrm{HCl}} \sim 200 \mathrm{ml} / \mathrm{min}$.

После завершения эпитаксиальных процессов были проведены эксперименты по травлению полученных образцов в растворе КОН. Для ex situ исследований морфологии поверхности выращенных образцов до и 
после травления использовался растровый электронный микроскоп (РЭМ) Zeiss Supra 25.

\section{3. Результаты и обсуждение}

Как уже было отмечено, МПЭ ПА синтез переходных слоев $\mathrm{GaN}$ на виртуальных подложках $\mathrm{SiC} / \mathrm{Si}(111)$ состоял из двух стадий: сначала выращивался зародышевый низкотемпературный слой $\mathrm{LT}-\mathrm{GaN}$ с толщиной $\sim 15 \mathrm{~nm}$ при $T_{S}=650^{\circ} \mathrm{C}$ и $F_{\mathrm{Ga}} / F_{\mathrm{N}} \sim 1$, после чего выращивался основной слой $\mathrm{GaN}$ с толщиной $\sim 750 \mathrm{~nm}$ при $T_{S}=730^{\circ} \mathrm{C}$ и $F_{\mathrm{Ga}} / F_{\mathrm{N}} \sim 6$. Следует отметить, что непосредственно перед началом роста LT-GaN наблюдалась „линейчатая“ картина ДОБЭ, свидетельствовавшая об относительно гладкой исходной поверхности $\mathrm{SiC}$. Вместе с тем, в процессе роста низкотемпературного зародышевого слоя $\mathrm{LT}-\mathrm{GaN}$ наблюдался постепенный переход к „точечной“ картине ДОБЭ, свидетельствующий о формировании слоя $\mathrm{LT}-\mathrm{GaN}$ с шероховатой морфологией поверхности. Однако, во время роста переходного слоя $\mathrm{GaN}$ при $T_{S}=730^{\circ} \mathrm{C}$ наблюдалось изменение „точечной“ картины ДОБЭ на „линейчатую“, которая свидетельствовала о двумерном росте переходного слоя $\mathrm{GaN}$.

После завершения процесса синтеза переходных слоев $\mathrm{GaN}$ методом МПЭ ПА полученный образец был разделен на три части. Одна часть была использована для идентификации полярности переходного слоя $\mathrm{GaN}$. Для этого выбранная часть образца была исследована с помощью РЭМ перед процессом травления в растворе $\mathrm{KOH}$. Проведенные предварительные РЭМ-исследования показали, что переходные слои $\mathrm{GaN}$, выращенные на виртуальных подложках $\mathrm{SiC} / \mathrm{Si}(111)$ имеют относительно гладкую поверхность. После этого исследованная часть образца была помещена в раствор $\mathrm{KOH}$, нагретый до $70^{\circ} \mathrm{C}$. РЭМ-исследования части образца, проведенные после процедуры травления, продемонстрировали, что выращенные на подложках $\mathrm{SiC} / \mathrm{Si}(111)$ методом МПЭ ПА переходные слои $\mathrm{GaN}$ характеризовались высокой скоростью травления в растворе $\mathrm{KOH}$, что позволило сделать вывод об N-полярности $\mathrm{GaN}$.

Таким образом, было установлено, что методом МПЭ ПА на поверхности виртуальной подложки $\mathrm{SiC} / \mathrm{Si}(111)$ был сформирован относительно гладкий $\mathrm{N}$-полярный переходный слой $\mathrm{GaN}$ с толщиной $\sim 800 \mathrm{~nm}$.

В следующей серии экспериментов был осуществлен ХГЭ-синтез основных слоев $\mathrm{GaN}$ на поверхностях, сформированных методом МПЭ ПА N-полярных переходных слоев $\mathrm{GaN}$. Как уже было отмечено, в одном из экспериментов рост основного слоя $\mathrm{GaN}$ осуществлялся методом ХГЭ непосредственно на поверхности $\mathrm{N}$-полярного переходного слоя $\mathrm{GaN}$. В то время как во втором ростовом процессе на поверхности $\mathrm{N}$-полярного переходного слоя $\mathrm{GaN}$ сначала методом ХГЭ был синтезирован промежуточный слой AlN c толщиной

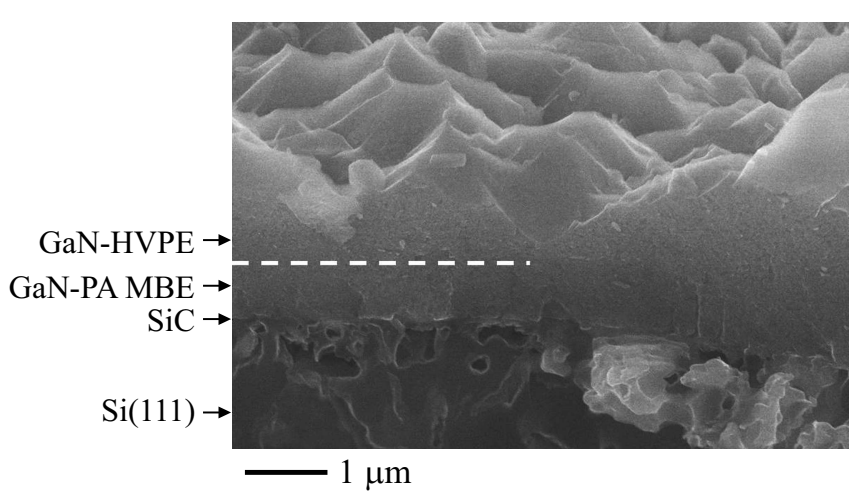

Рис. 1. РЭМ-изображения образца, в котором основной слой $\mathrm{GaN}$ был выращен методом ХГЭ непосредственно на поверхности $\mathrm{N}$-полярного переходного слоя $\mathrm{GaN}$, выращенного методом МПЭ ПА на подложке $\mathrm{SiC} / \mathrm{Si}(111)$.

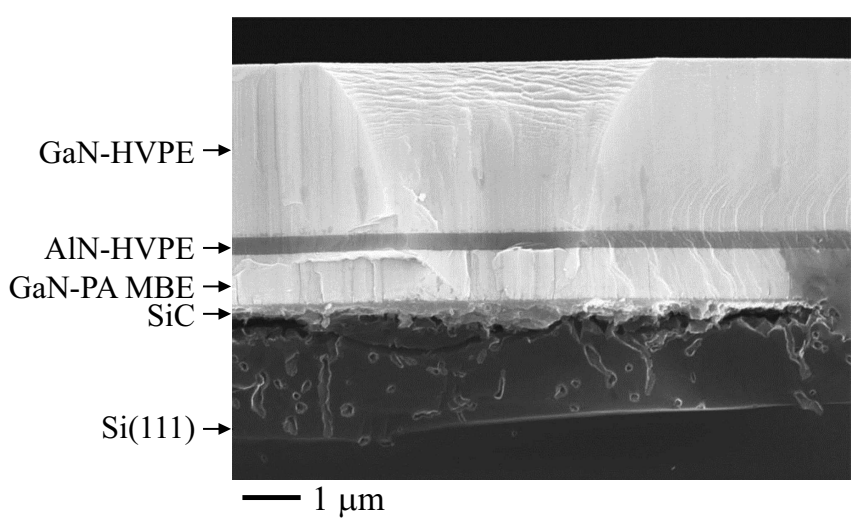

Рис. 2. РЭМ-изображения образца, выращенного с использованием промежуточного слоя AlN, синтезированного методом ХГЭ на поверхности $\mathrm{N}$-полярного переходного слоя GaN непосредственно перед ХГЭ синтезом основного слоя $\mathrm{GaN}$.

$\sim 280 \mathrm{~nm}$, после которого был выращен основной слой $\mathrm{GaN}$ с толщиной $\sim 3 \mu \mathrm{m}$.

Исследования синтезированных образцов с помощью РЭМ показали, что в случае ХГЭ синтеза GaN непосредственно на поверхности $\mathrm{N}$-полярного переходного слоя $\mathrm{GaN}$, без использования $\mathrm{AlN}$ промежуточного слоя, формировался поликристаллический слой $\mathrm{GaN}$ (см. рис. 1). Вместе с тем, РЭМ-исследования образца, выращенного с использованием промежуточного слоя $\mathrm{AlN}$, который был синтезирован методом ХГЭ на поверхности $\mathrm{N}$-полярного переходного слоя $\mathrm{GaN}$ непосредственно перед ХГЭ синтезом основного слоя $\mathrm{GaN}$, свидетельствовали об относительно гладкой морфологии поверхности основного слоя $\mathrm{GaN}$ (см. рис. 2). Кроме того, РЭМ-изображения данной многослойной гетероструктуры GaN/AlN/GaN/SiC/Si(111) демонстрировали однородность и резкость гетерограниц, а также отсутствие микротрещин.

После РЭМ-исследований морфологии поверхности выращенной гетроструктуры GaN/AlN/GaN/SiC/Si(111) 


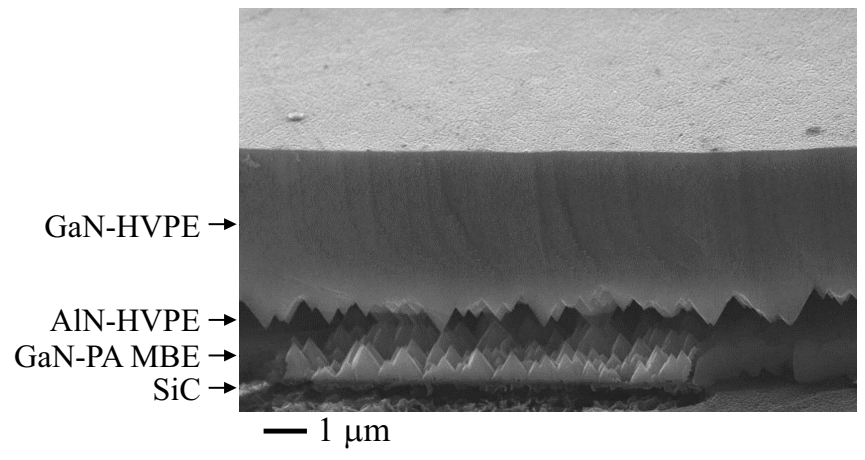

Рис. 3. РЭМ-изображения образца, выращенного с использованием промежуточного слоя AlN, синтезированного методом ХГЭ на поверхности $\mathrm{N}$-полярного переходного слоя $\mathrm{GaN}$ непосредственно перед ХГЭ синтезом основного слоя $\mathrm{GaN}$, полученные после травления в растворе $\mathrm{KOH}$.

были проведены исследования ее полярности. Для этого выращенная гетероструктура была помещена в раствор $\mathrm{KOH}$, нагретый до $70^{\circ} \mathrm{C}$, после чего были проведены повторные РЭМ-исследования ее морфологии, которые показали отсутствие следов травления основного слоя $\mathrm{GaN}$, выращенного ХГЭ, что свидетельствовало о его $\mathrm{Ga}$-полярности. B тоже время, как и предполагалось, $\mathrm{N}$-полярный переходный слой $\mathrm{GaN}$, выращенный методом МПЭ ПА на подложке $\mathrm{SiC} / \mathrm{Si}(111)$, был практически полностью удален после 10 min травления гетроструктуры $\mathrm{GaN} / \mathrm{AlN} / \mathrm{GaN} / \mathrm{SiC} / \mathrm{Si}(111)$ в растворе $\mathrm{KOH}$, что привело к отделению основного слоя $\mathrm{GaN}$ от подложки (рис. 3). Вместе с тем, на РЭМ-изображениях, представленных на рис. 3 также наблюдается травление промежуточного слоя AlN, начинающееся с его тыльной стороны, то есть со стороны гетрограницы между AlN и N-полярным переходным слоем $\mathrm{GaN}$. Таким образом, можно предположить, что в выращенной гетероструктуре $\mathrm{GaN} / \mathrm{AlN} / \mathrm{GaN} / \mathrm{SiC} / \mathrm{Si}(111)$ промежуточный слой AlN и основной слой $\mathrm{GaN}$, выращенные методом ХГЭ на N-полярном переходном слое $\mathrm{GaN}$, имели $\mathrm{Ga}(\mathrm{Al})$-полярность.

Природа обнаруженного нами эффекта инверсии полярности пока не ясна. Для ее объяснения будут проведены дополнительные исследования. В данной работе мы не будем излагать гипотезы, которые не имеют строгого подтверждения. В данном случае важен факт, что верхний, основной слой $\mathrm{GaN}$, выращенный методом ХГЭ на слое AlN наследует металлическую полярность и, соответственно, практически не подвержен травлению в $\mathrm{KOH}$

\section{4. Заключение}

В работе продемонстрировано, что N-полярные переходные слои $\mathrm{GaN}$, синтезированные методом МПЭ ПА на виртуальных подложках $\mathrm{SiC} / \mathrm{Si}(111)$ могут быть использованы в качестве переходных, буферных слоев для последующего роста методом ХГЭ свободных от трещин основных слоев $\mathrm{GaN}$ с толщиной вплоть до $3 \mu \mathrm{m}$. Экспериментально установлено, что для ХГЭ-синтеза относительно гладких основных слоев $\mathrm{GaN}$ на поверхности $\mathrm{N}$-полярного переходного слоя $\mathrm{GaN}$ предварительно методом ХГЭ необходимо сформировать промежуточный слой $\mathrm{AlN}$ с толщиной $\sim 280 \mathrm{~nm}$. Установлено, что промежуточный слой $\mathrm{AlN}$ и основной слой $\mathrm{GaN}$, выращенные методом ХГЭ на N-полярном переходном слое $\mathrm{GaN}$, имеют $\mathrm{Al}(\mathrm{Ga})$-полярность. Установлено, что травление гетероструктуры GaN/AlN/GaN/SiC/Si(111) с переменной полярностью, синтезированной с использованием интеграции технологий МПЭ ПА и ХГЭ, в растворе $\mathrm{KOH}$ приводит к практически полному удалению $\mathrm{N}$-полярного переходного слоя $\mathrm{GaN}$ и отделению основного слоя $\mathrm{GaN}$ от подложки $\mathrm{SiC} / \mathrm{Si}(111)$.

\section{Финансирование работы}

Эксперименты по МПЭ ПА синтезу переходных слоев $\mathrm{GaN}$ проводились в рамках выполнения государственного задания министерства образования и науки Российской Федерации № 16.9789.2017/БЧ. Морфологические исследования образцов выполнены в рамках генерального соглашения о научно-исследовательской деятельности между Сколтехом и СПбАУ РАН (№ 3663-MRА, проект 4). Синтез структур $\mathrm{SiC} / \mathrm{Si}$, рост пленок $\mathrm{AlN}$ и $\mathrm{GaN}$ метолом ХГЭ финансировался в рамках гранта РНФ № 19-72-30004.

А.М. Мизеров, С.Н. Тимошнев, К.Ю. Шубина, Т.Н. Березовская, Д.В. Мохов и А.Д. Буравлев выполняли свою часть работы в рамках государственного задания министерства образования и науки Российской Федерации № 16.9789.2017/БЧ, генерального соглашения о научно-исследовательской деятельности между Сколтехом и Академическим университетом (№ 3663-MRA, проект 4). А.В. Осипов выполнял свою часть работы в рамках гранта РНФ № 19-72-30004.

\section{Конфликт интересов}

Авторы заявляют, что у них нет конфликта интересов.

\section{Список литературы}

[1] K. Kim, M. Hua, D. Liu, J. Kim, K.J. Chen, Z. Ma. Nano Energy 43, 259 (2018).

[2] R. Hentschel, J. Gärtner, A. Wachowiak, A. Großer, T. Mikolajick, S. Schmult. J. Crystal Growth 500, 1 (2018).

[3] A. Binder, J.-S. Yuan, B. Krishnan, P.M. Shea. Superlat. Microstruct. 121, 92 (2018).

[4] G.H. Chung, T.A. Vuong, H. Kim. Results Phys. 12, 83 (2019).

[5] Y. Li, X. Xiu, Z. Xiong, X. Hua, Z. Xie, T. Tao, P. Chen, B. Liu, R. Zhang, Y. Zheng. Mater. Lett. 240, 121 (2019).

[6] J.A. Freitas Jr., J.C. Culbertson, N.A. Mahadik, M.J. Tadjer, S. Wu, B. Raghothamachar, M. Dudley, T. Sochacki, M. Bockowski. J. Crystal Growth 500, 104 (2018). 
[7] N. Zainal, M.E.A. Samsudin, M.I.M. Taib, M.A. Ahmad, A. Ariff, N. Alwadai, I.S. Roqan. Mater. Sci. Semiconduct. Proc. 88, 40 (2018).

[8] T.H. Yang, J.T. Ku, J.-R. Chang, S.-G. Shen, Y.-C. Chen, Y.Y. Wong, W.C. Chou, C.-Y. Chen, C.-Y. Chang. J. Crystal Growth 311, 1997 (2009).

[9] А.М. Мизеров, С.Н. Тимошнев, М.С. Соболев, Е.В. Никитина, К.Ю. Шубина, Т.Н. Березовская, И.В. Штром, А.Д. Буравлев. ФТП 52, 1425 (2018).

[10] S.A. Kukushkin, A.M. Mizerov, A.V. Osipov, A.V. Redkov, S.N. Timoshnev. Thin Solid Films 646, 158 (2018).

[11] С.А. Кукушкин, А.М. Мизеров, А.С. Гращенко, А.В. Осипов, Е.В. Никитина, С.Н. Тимошнев, А.Д. Буравлев, М.С. Соболев. ФТП 53, 190 (2019).

[12] S.A. Kukushkin, Sh.Sh. Sharofidinov, A.V. Osipov, A.V. Redkov, V.V. Kidalov, A.S. Grashchenko, I.P. Soshnikov, A.F. Dyadenchuk. ECS J. Solid State Sci. Technology 8, X1 (2019).

[13] С.А. Кукушкин, А.В. Осипов. ФТТ 50, 1188 (2008).

[14] С.А. Кукушкин, А.В. Осипов. ДАН 444, 266 (2012).

[15] С.А. Кукушкин, А.В. Осипов. Изв. РАН. МТТ 2, 122 (2013).

[16] С.А. Кукушкин, А.В. Осипов, Н.А. Феоктистов. ФТТ 56, 1457 (2014).

Редактор К.В. Емщев 